\title{
Combined release of antiseptic and antibiotic drugs from visible light polymerized biodegradable nanocomposite hydrogels for periodontitis treatment
}

\author{
József Bakó \\ University of Debrecen \\ Ferenc Tóth \\ University of Debrecen \\ József Gáll \\ University of Debrecen \\ István Varga \\ University of Debrecen \\ Anton Sculean \\ University of Bern
}

Romána Zelkó

Semmelweis University

Csaba Hegedũs ( $\square$ hegedus.csaba.prof@dental.unideb.hu )

University of Debrecen

\section{Research Article}

Keywords: periodontal therapy, biodegradable nanocomposite hydrogel, $\mathrm{pH}$ changes

Posted Date: October 4th, 2021

DOl: https://doi.org/10.21203/rs.3.rs-955354/v1

License: (c) (1) This work is licensed under a Creative Commons Attribution 4.0 International License. Read Full License 


\title{
Combined release of antiseptic and antibiotic drugs from visible light polymerized biodegradable nanocomposite hydrogels for periodontitis treatment
}

\author{
József Bakó1,* ${ }^{*}$ Ferenc Tóth ${ }^{1}$, József Gáll ${ }^{2}$, István Varga ${ }^{3}$, Anton Sculean ${ }^{4}$, Romána Zelkó ${ }^{5}$, \\ and Csaba Hegedús ${ }^{1}$
}

\author{
${ }^{1}$ University of Debrecen, Faculty of Dentistry, Department of Biomaterials and Prosthetic Dentistry, Debrecen, 4028, \\ Hungary \\ ${ }^{2}$ University of Debrecen, Faculty of Informatics, Department of Applied Mathematics and Probability Theory, \\ Debrecen, 4028, Hungary \\ ${ }^{3}$ University of Debrecen, Faculty of Dentistry, Department of Periodontology, Debrecen, 4028, Hungary \\ ${ }^{4}$ University of Bern, School of Dental Medicine, Department of Periodontology, Bern, 3010, Switzerland \\ ${ }^{5}$ Semmelweis University, Faculty of Pharmaceutical Sciences, Department of Pharmacy Administration, Budapest, \\ 1085, Hungary \\ *bako.jozsef@dental.unideb.hu
}

\begin{abstract}
The in-situ used combination of different types of drugs revolutionized the area of periodontal therapy. The purpose of this study was to develop biodegradable nanocomposite hydrogel $(\mathrm{NCHG}$ ) as a pH-sensitive drug delivery system. To reach the local applicability of the NCHG in dental practice routinely used blue-light photopolymerization was chosen for the preparation. The reaction time was 60 seconds, which resulted in stable hydrogel structures. Universal Britton-Robinson buffer solutions were used to investigate only the effect of $\mathrm{pH}$ for the release of the periodontal pocket useable drugs in the 4-12 range. The release of metronidazole was faster, it came out from the NCHGs within 12 hours, but the chlorhexidine had shown a longer elution time, which was more than 7 days as far as the antiseptic effect was maintained. The release of chlorhexidine showed strong $\mathrm{pH}$ depending. The biocompatibility of the NCHGs was proved by Alamar-blue test, and the effectiveness of drug release in the acidic medium was demonstrated. This fast photopolymerizable NCHG can help to approach an ideal locally useable combined drug delivery system which can be used in the amount required of the medicines and can reduce the side effects with the increased effectiveness by following $\mathrm{pH}$ changes.
\end{abstract}

\section{Introduction}

Periodontitis is defined as an inflammatory disease of the periodontium that concerns about $10-15 \%$ of the world population ${ }^{1}$. The combined drug delivery systems are more and more frequently investigated areas in medical fields, thanks to the success of different antibiotic therapies and anti-cancer therapies. The enhancement of the effectiveness and the possibility of the decrease of the applicable dose of drugs are desirable properties and appropriate directions for the developments. The not well-established usage of antibiotics can lead to the progression of resistance. Dual or triple usage of antibiotic combinations can be more effective in treatment ${ }^{2,3}$. Besides the combination of the antibiotics, another way can be for the increase of effectiveness the direct local usage. The effectiveness of the applied drugs can be up to 100-fold higher administered in the therapeutic doses compared with the systemic usage ${ }^{4,5}$.

The applicable doses of drugs are greatly reduced when the treatment is performed only in the affected area ${ }^{6,7}$. In the field of dentistry, periodontitis is a chronic inflammatory disease triggered by periodontal pathogenic bacteria which can result in loss of tooth's supporting bone and fiber apparatus, formation of periodontal pockets ultimately leading to tooth $\operatorname{loss}^{8,9}$. Since deep periodontal pockets ensure an ideal environment for the proliferation of pathogenic microorganisms, the local administration of antibiotics can be an ideal form of treatment because high therapeutic doses can be reached by significantly less drug quantities, thus leading to fewer side effects ${ }^{10}$. However, in order to enable adequate effectiveness of the drugs, the delivery systems must cover the affected area (i.e. the periodontal pocket), ensuring that the necessary concentration is obtained and maintained until the desired microbiological and clinical effects are reached ${ }^{11}$.

In dental practice, the application of visible (blue) light photopolymerization is general; it follows that this can be one of the most convenient ways for the construction of locally useable drug delivery devices and can open a gate to the adaption of this 
system to the 3D printing technology. The polymer system can fill into space next to the tooth (e.g.: into a periodontal pocket) and after the fast polymerization, the stable crosslinked structure can release the embedded drugs during a longer period. The biopolymer-based hydrophilic systems give a wide range of opportunities, for the creation of local applicable systems, because of versatile forms e.g.: gels, hydrogels, nanogels, fibers, strips, or chips. The combination of these forms can optimize the necessary properties, from the mechanical to the chemical and physical behavior. The poly- $\gamma$-glutamic acid is water-soluble, in the aqueous phase in a negatively charged biopolymer. Biocompatible, biodegradable, and edible polymer, with various modification possibilities ${ }^{12}$. The methacryloil-group modified (MPGA) polymer form and an earlier created nanoparticle (PGA-MNP) version can work together as a special composite with the possibilities of the release of the different drugs at different rates ${ }^{13,14}$. The surface volume ratio of nanoparticles ensures the chance for adjusting the release rate of one of the active components, while another drug can comes out from the matrix in an environment-dependent way. The combination of antiseptic and antibiotic drugs can ensure a broad spectrum of the medical effect and helps in faster healing with fewer side effects.

Periodontal inflammations are polymicrobial infections and periodontitis is diagnosed clinically instead of a microbiological investigation. As a result, using adjunctive antibiotics in periodontitis has tended to be empirical. Antibiotics must be used as an adjunct to root surface debridement and mechanical destruction of the biofilm. These adjunctive treatments can enhance the outcomes of mechanical treatment ${ }^{15}$. Systemic antibiotics may be used in patients who have stage 4 grade $\mathrm{C}$ periodontitis, multiple periodontal abscesses, and necrotizing gingivitis/periodontitis. Monotherapies and combination therapies are available. The most effective antibiotics are amoxicillin and metronidazole ${ }^{15}$. Topical antimicrobials are introduced to minimize systemic side effects and avoid problems with patient compliance ${ }^{16}$. To complement phase I periodontal therapy, there are multiple options of antimicrobial agents that can be locally delivered, such as metronidazole, chlorhexidine, minocycline, doxycycline, and tetracycline. However, an important aspect is that all antibiotics must meet the following criteria: the drug must easily reach the targeted area and remain at an effective concentration, furthermore, it should last for a long period of time ${ }^{17}$.

The different stimuli-responsive drug delivery systems can ensure the benefit of the natural controlling of the inflammatory processes when $\mathrm{pH}$ is decreased. Many periodontal systems aim at using the advantage of this possibility and therefore, different polymeric systems and mesoporous silica-based drug releasing materials were created and investigated in the last decade. These materials and combinations show better control possibilities and more flexible availability of the drugs directly in time at the place of application $^{18,19}$.

Metronidazole (Metr) is a well-known treatment possibility for periodontal diseases. It is available in different forms and some developments can be found which investigated the effectiveness of metronidazole alone or as a part of the delivery system $^{20,21}$. Chlorhexidine $(\mathrm{CHX})$ is a generally applied antiseptic agent that can be used as a "gold standard" during investigations of new drugs or delivery systems. The effectiveness and applicability of this drug are well based in the oral region because of a wide range of antimicrobial effects and adhesive properties ${ }^{22,23}$. In our earlier study we investigated individually the releases of antibiotic drugs from the hydro- and the nano-gels, but the combination of the different forms of the polymers, and the different drugs can give more specialized treatment possibilities in general, and particularly in the field of dentistry. The aim of this work was to demonstrate an MPGA/PGA-MNP created blue-light photo-polymerizable nanocomposite hydrogel (NCHG), and the investigations of the release profiles of the $\mathrm{CHX}$ and Metr drugs on different pH in a universal Britton-Robinson buffer solution.

\section{Results and discussion}

\section{Modifications of MPGA}

\section{Characterization of the methacrylated components}

The methacrylation reactions of PGA were successful as the signals show in Figures 1 and 2. The signals of chemical shifts of methacryloyl-groups were assigned $\delta=6.09,5.70$, and $1.88 \mathrm{ppm}$. The rate of the methacrylation could not reach the theoretical level $(50 \%)$ but these materials as parts of the composite were able to react in a fast photo-crosslinking reaction within 60 sec, and with this amount of methacryloyl-groups it can be swollen in water. The decreased methacrylation rate is unfamiliar with PGA Zheng et al find a similar effect in a comparable reaction ${ }^{24}$.

The evidence of the success of the crosslinking reaction was performed by the assignments of the signals of crosslinker moiety from EDA $\delta=3.23,3.62,3.70 \mathrm{ppm}\left(\mathrm{CH}_{2}\right.$ groups). The methacryloyl and crosslinker signals in Figures 1 and 2 represent, that the chemical modifications were done in the backbone of the PGA molecule, and photopolymerizable MPGA polymer and PGA-MNPs were created.

\section{NCHG preparation}

The schematic way of the preparation of NCHGs is presented in Figure 3. The total polymer content is $33.3 \mathrm{w} / \mathrm{w} \%$, in which 2/3 part MPGA and 1/3 part PGA-MNPs were mixed with Metr and CHX solutions, respectively. The photo initiator was added to the complete mixture and after homogenization $60 \mathrm{sec}$ photopolymerisation occurred on every sample. 


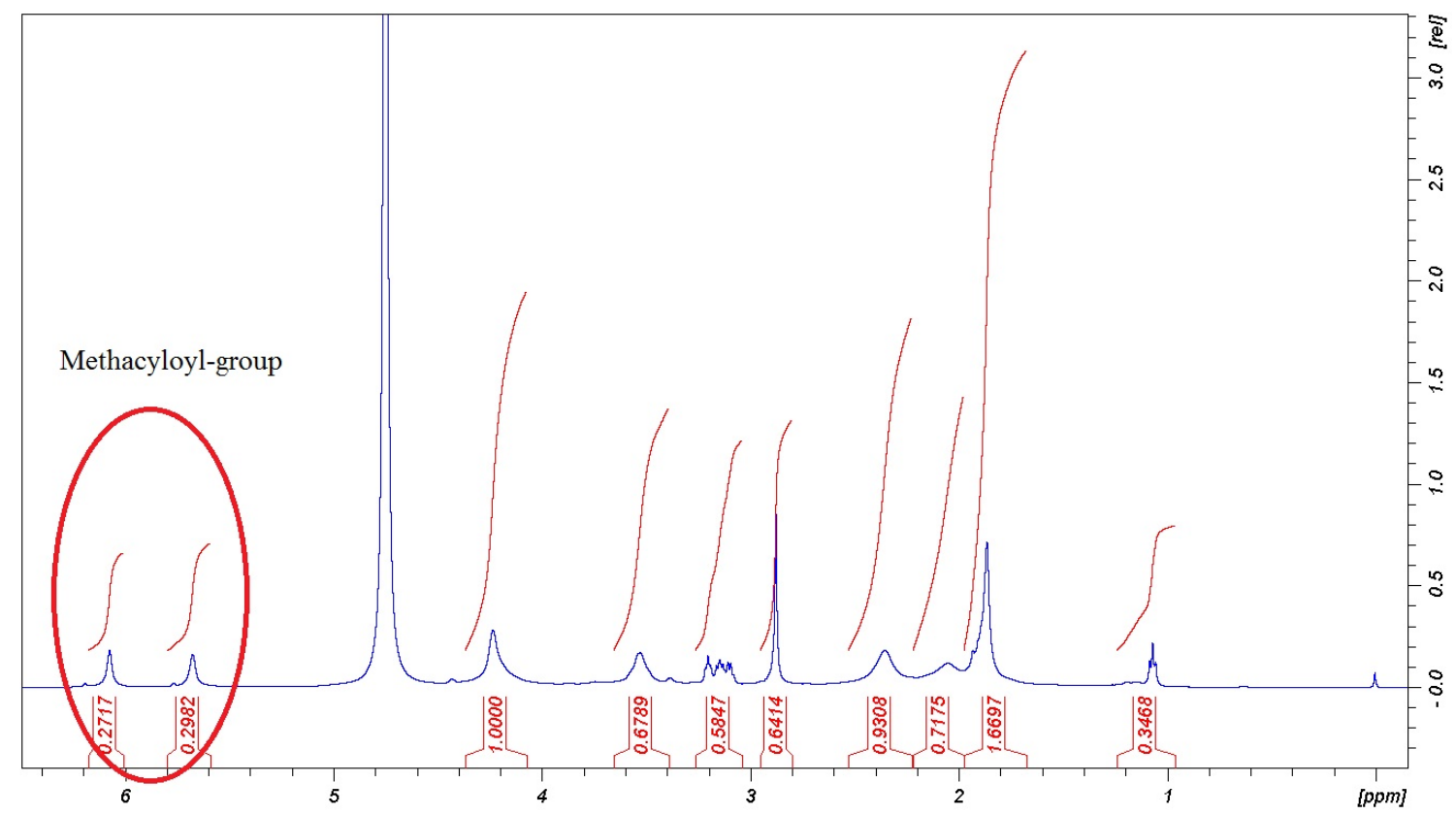

Figure 1. ${ }^{1} \mathrm{H}-\mathrm{NMR}$ spectra of MPGA polymer

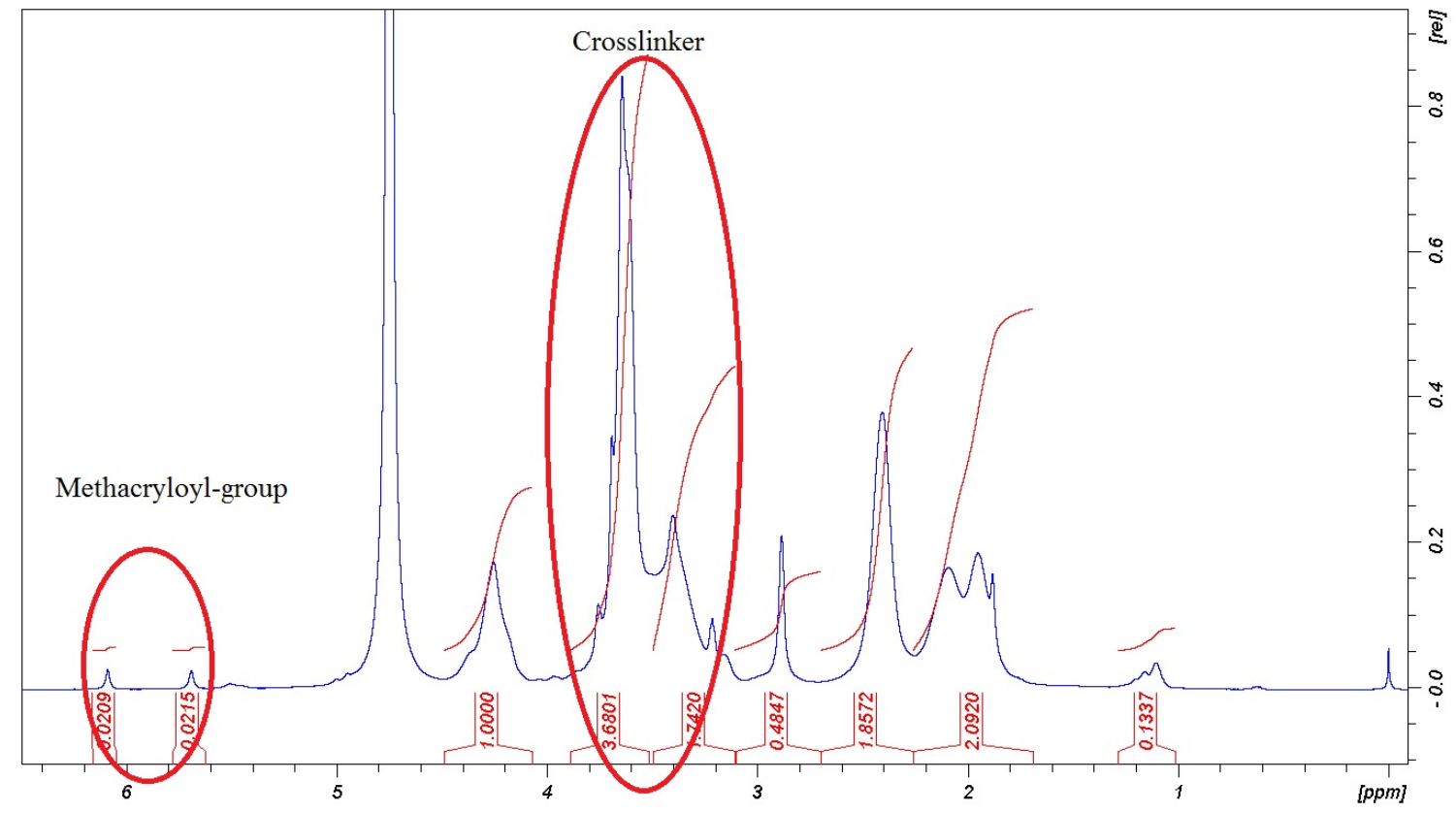

Figure 2. Figure 2. ${ }^{1} \mathrm{H}-\mathrm{NMR}$ spectra of PGA-MNP polymer 


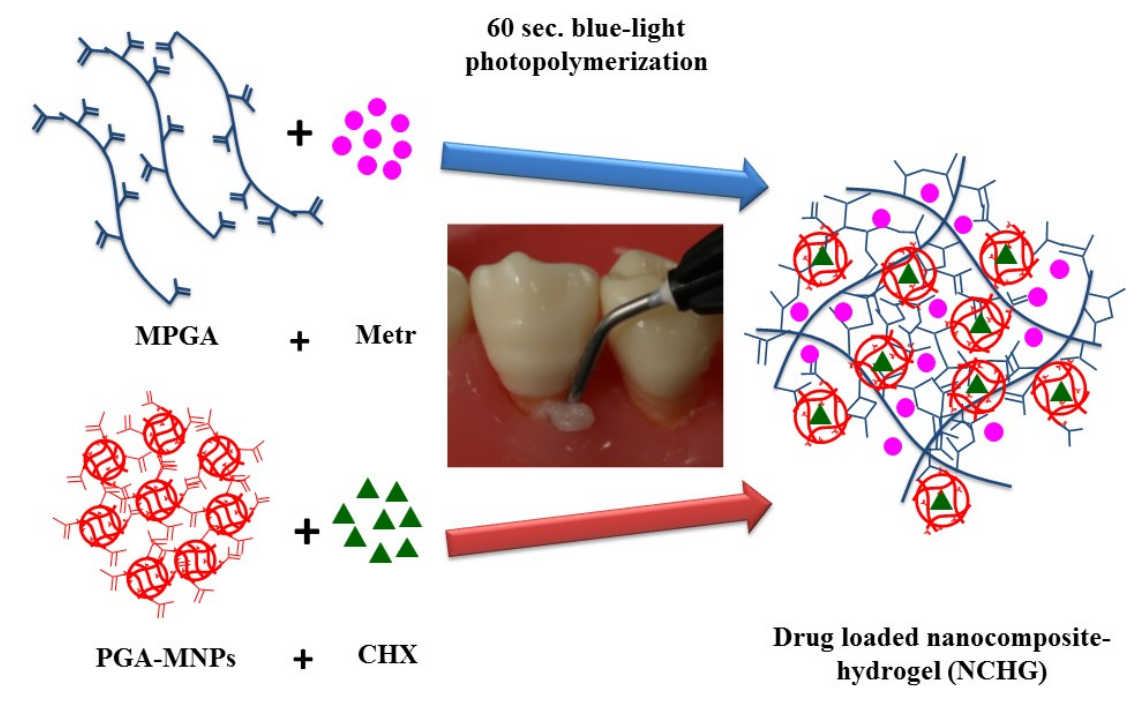

Figure 3. Schematic way of the preparation of MPGA/PGA-NPs nanocomposite hydrogels

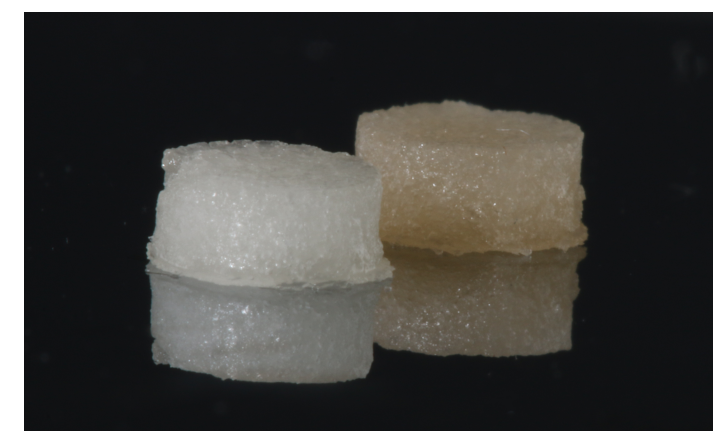

Figure 4. Visible-light photopolymerized NCHGs

\section{Characterization of the MPGA/PGA-NP nanocomposite hydrogel Mechanical investigations}

The mechanical properties of the NCHGs ensure the evidence that the methacryloyl-groups were reactive and the MPGA matrix forming and PGA-MNPs as nano-components were able to form physically stable hydrogels after 60 sec exposure of an everyday used in dental practice hand lamp. The picture of these gels are shown in Figure 4. These results could provide a promising basis for further developments, so that this system can be applied to new 3D printing technologies with only minor adaptations.

The results of mechanical investigations shown (Table 1), that the drug-containing NCHGs are not as strong as the polymer composition without active ingredients. The $0.1094 \mathrm{MPa}$ compressive stress and the $0.2237 \mathrm{MPa}$ Young-modulus next to the $0.5780 \mathrm{~mm} / \mathrm{mm}$ strain values were particularly promising because these parameters mean that these polymer systems can work properly as drug delivery systems. These parameters mean that this structure can withstand the forces of the soft tissues next to the teeth ${ }^{13,25}$. The polymer composition without drugs shown much higher compressive stress $0.2924 \mathrm{MPa}$, and Young-modulus $2.3845 \mathrm{MPa}$ values and less elastic properties with the 0.2224 strain, which means that the filling of this system with any other bioactive components could be able to fulfill the requirements of drug delivery systems and might provide useful tools for other biomedical fields such as tissue engineering. In all cases (Young modulus, load, stress, strain) the control group (NCHG without drugs) and the NCHG filled with drugs showed clearly a significant difference of means by the $t$ tests as well as the Mann-Whitney tests $(\mathrm{P}<0.001)$. These mechanical parameters show similar or higher values than others on the biomedical 


\begin{tabular}{|c|c|c|c|c|}
\hline n=min. 9 & \multicolumn{2}{|c|}{ Control (NCHG) } & \multicolumn{2}{c|}{ NCHG+CHX+Metr } \\
\hline & Mean & SD & Mean & SD \\
\hline \hline Young modulus (MPa) & 2.3845 & 0.3594 & 0.2237 & 0.0757 \\
Compressive strain (mm/mm) & 0.2224 & 0.0251 & 0.5800 & 0.0733 \\
Compressive load (N) & 4.8806 & 0.1300 & 1.8458 & 0.6466 \\
Compressive stress (MPa) & 0.2924 & 0.0178 & 0.1094 & 0.0340 \\
\hline
\end{tabular}

Table 1. Mechanical properties of nanocomposite hydrogels

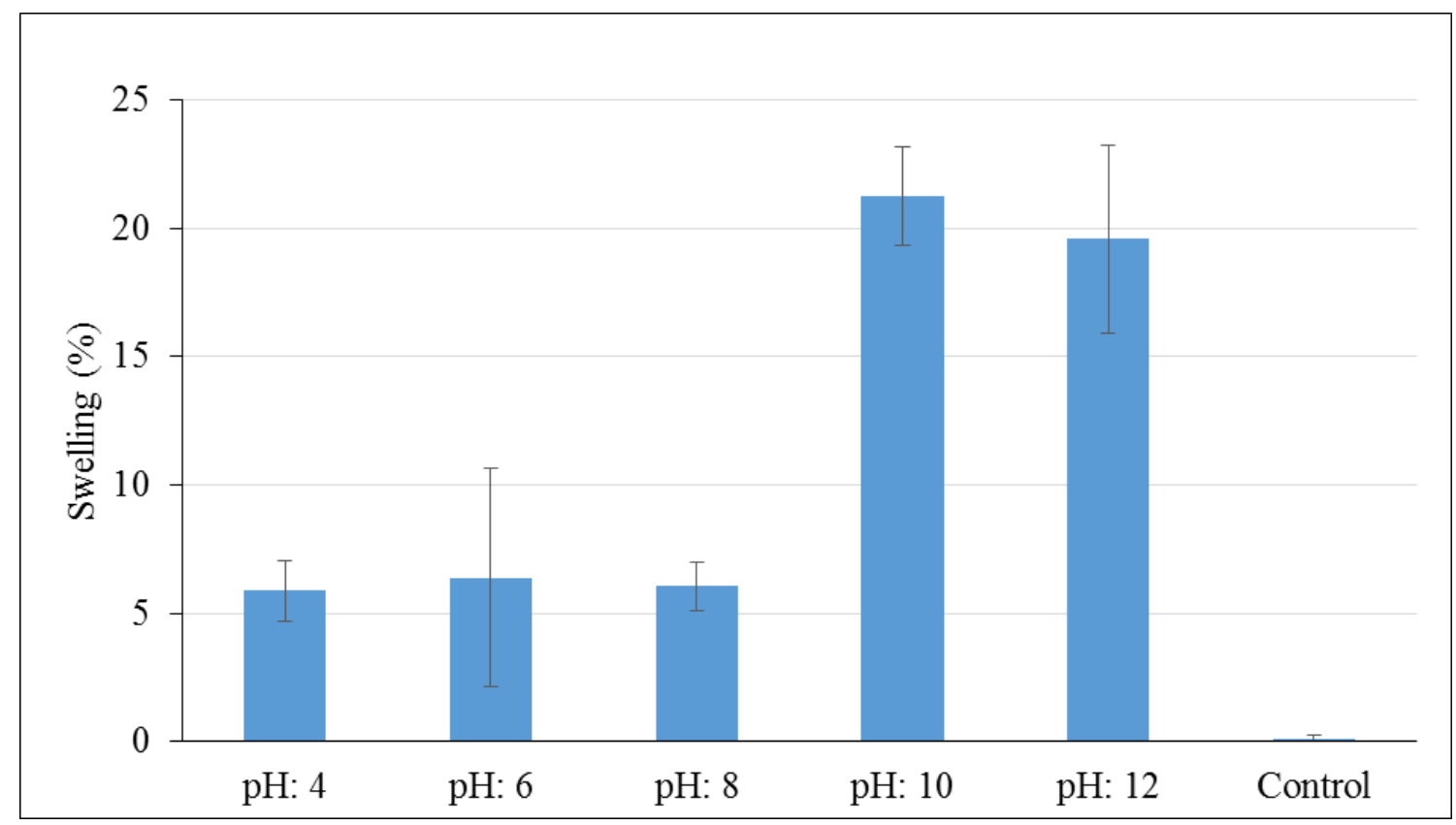

Figure 5. Swelling properties of CHX/Metr loaded NCHGs

In different $\mathrm{pH}$ Britton-Robinson buffer solutions after 168 hours. Control is the NCHG structure without drugs, error bars represent the standard deviation (SD) of five parallel measurements.

field composed systems that varied from a few tens to around $100 \mathrm{kPa}$ compressive stress ${ }^{24}$.

\section{Swelling properties}

The results of the swelling properties showed that the volume of the NCHGs did not change notably, but depending on the $\mathrm{pH}$ the growth was around $5 \%$ in acidic or around $20 \%$ in the alkaline medium after one week (Figure 5). The statistical analysis proved that the linear models gave better fit to the swelling measures $(\mathrm{R}=0.810)$ than the fitted non-linear ones (exponential, power). Note, that the choice of the best curve was not our main goal, one could make further selection of non-linear models on a larger data set. Importantly, the swelling shows a significant positive relationship with the $\mathrm{pH}$ value (coefficient $=2.112, \mathrm{P}<$ 0.001 ) as it was expected. Naturally, the control sample without drugs showed the lowest swelling result which is substantially different from the weight increasing of drug-containing NCHGs on other pHs. These are promising results, which suggest that in the condition of application -in an acidic medium- the gels can fill the necessary volume, but it will not grow out from the available spaces. These findings were essential because in some of the biopolymer-based systems especially with the presence of PGA swelling behavior could be higher sometimes more than 200 or $300 \%{ }^{26}$.

\section{Study of drug release properties on different $\mathrm{pH}$}

The release properties of the NCHG have shown that the different $\mathrm{pH}$ of the medium affected the release of the different drugs to varying degrees. The Metr was not substantially affected by the $\mathrm{pH}$, and this drug was released from the composite in the early period of the study. Figure 6 shows that this period is around 8-12 hours, which means much longer antibiotic present than the usage of a drug solution. In Figure 7(A), where the cumulative amounts of released Metr was represented as a function of the complete drug content, this leaching out phenomena is recognized, but this period can be longer in practice next to the 
tooth in a pocket where only a small amount of sulcus fluid is flowing slowly. This effect can ensure the continuous presence of the antibiotic drug in around half a day what can be essential, and sufficient for the fast decrease of the number of the bacteria such as Actinobacillus actinomycetemcomitans, Porphyromonas gingivalis, Prevotella intermedia ${ }^{27,28}$. After this period, the effect of CHX can control the reproduction of the rest of the bacteria. Namely, the $\mathrm{pH}$ of the medium had a great influence on the release of the $\mathrm{CHX}$, and especially in the case of $\mathrm{pH} \mathrm{4,} \mathrm{where} \mathrm{the} \mathrm{concentration} \mathrm{of} \mathrm{the} \mathrm{drug} \mathrm{was} \mathrm{continuously} \mathrm{above}$ the clinical effective concentration ${ }^{29,30}$. The total released drug concentrations showed that the entire amount of Metr was liberated around 8-12 hours from the nanocomposite hydrogels. This effect is very important because it can eliminate the high pathogen presence, and this time period is substantially longer than in a case of simple irrigation with the solution of the drug. The release tendency of the $\mathrm{CHX}$ shows strong $\mathrm{pH}$ dependence, the liberation of $\mathrm{CHX}$ is increasing with the acidity of the media (Figure 6 and 7(B)). It is noticeable that the acidic $\mathrm{pH}$-in this case the $\mathrm{pH} 4$ - showed the most substantial difference compared to all other $\mathrm{pH}$ and provided the best releasing results. In a statistical analysis of cumulative release of results Metr and $\mathrm{CHX}$ after the logit transform we could fit on both variables -as dependent ones- linear regression models with random effect (controlling for the different series of experiments), where time (hours) and $\mathrm{pH}$ play the role of a significant independent variable in both models. Namely, (i) for the Metr case time has a positive relationship with release $($ coefficient $=0.0102, \mathrm{P}<$ 0.001 ), whereas larger $\mathrm{pH}$ values go together with smaller release measures (coefficient $=-0.1261$, $\mathrm{P}=0.025$ ), being everything else the same; secondly, (ii) for the $\mathrm{CHX}$ case time has a positive relationship with release $($ coefficient $=0.0106, \mathrm{P}<0.001)$, whereas larger $\mathrm{pH}$ values go together with smaller release measures (coefficient $=-0.2666, \mathrm{P}<0.001$ ). After one week the half of the embedded CHX comes out from the NCHGs, but Figure 8 gives some evidence that all of CHX could be available in the appropriate conditions, because at $\mathrm{pH} 2$ almost the entire amount of the drug was released from the NCHGs, and the usage of the enzymes could not significantly alter the process. The photopolymerization reactions of the NCHGs or the absorbability of the CHX do not cause any decomposition of the drug and allow the continuous release of the active ingredient. In this way, we can reach an antiseptic effect locally in a long term, and do not have to count with the side effect of CHX used in higher concentration e.g.: discoloration of teeth or filling, or bitter taste sensation ${ }^{31}$. The presence of CHX can decrease the activity of the metalloproteinases 2, 8, and 9 of $P$. gingivalis, and reduce the adhesion rate so it is effective in an inflammatory reaction ${ }^{32,33}$. This drug should be more frequently used in periodontal therapy, especially when directly applied in the periodontal pockets ${ }^{34}$. Thus, the locally used antibiotic, and antiseptic intra-pocket drug delivery system ensure a higher concentration of active content in the gingival crevice fluid therefore advanced effectiveness, and better patient compliance could be achieved ${ }^{35}$. Different locally used drug delivery systems are developed from the strips, fibers, or microparticles, but next to the opportunities stay deficiencies into the individual types ${ }^{36-38}$. The local administrations provide benefits as the less drug needed but the usage of the appropriate dose is often a challenge in a real situation ${ }^{39}$. The injectable systems can give more flexibility in this regard, but the localization of this drug containing polymers for a necessarily long time can be a remaining difficulty ${ }^{40,41}$. The hydrogels have versatile crosslinking possibilities from the softer physical interactions to the stronger chemical bonding formations create any chances to control the stability in time with the presence of drug but some more sensitive agents cannot be active after harder initiation processes like UV-light or higher temperature ${ }^{42}$. Recently, the stimuli-responsive systems target at bringing closer the possibilities and the aims e.g.: the $\mathrm{pH}$-dependent drug delivery devices can provide one the most appropriate results in an inflammatory reaction, wound healing, or tissue engineering ${ }^{43,44}$.

Until today, several studies have shown a change in the release of $\mathrm{CHX}$ as a result of $\mathrm{pH}$ change, and more articles can be found as examples for the $\mathrm{pH}$-dependent properties of the PGA, but the combination of these systems can provide the base of novel treatment concepts for patients with moderate or severe periodontitis ${ }^{6,42,45,46}$. The combination of drugs to increase the effectiveness and decrease the necessary amount of the applied medicines is a long-standing endeavor. Aspirin and erythropoietin-filled locally used hydrogels were demonstrated for the treatment of periodontal disease, and another recent example studied the combined application of CHX and ibuprofen in intra-pockets administration and proved their clinical relevance $^{47,48}$. Our newly created CHX/Metr containing NCHG system is novel, and clinically promising, evidenced by the half-day presence of the antibiotics, and the, at least one week long-lasting, antimicrobial environment. The practical applicability is facilitated by the really fast one-minute visible-light polymerization reaction - directly used by a dental-hand lamp blue-light - and the easy administration possibilities.

\section{Cell viability assay}

MG63 cells were cultured in the presence or absence of CHX and Metr containing NCHG samples and cell viability was determined by Alamar Blue assay after 1, 3, and 7 days, and media was changed after each measurement.

After 1 day of incubation, the cell viability was slightly but statistically significantly $(\mathrm{P}=0.0406)$ reduced in the presence of the CHX and Metr containing NCHG samples (Figure 9) compared to the control. However, at the following examination days, this reduction disappeared completely and no changes in the viability of the cells cultured in the presence of the hydrogel sample compared to the control were detected. This observation suggests, that most of the unreacted monomers which can cause a reduction in the cell viability were released from the hydrogels on the first day and after the addition of fresh medium, it could not reach an effective concentration to cause a further reduction in the viability. 


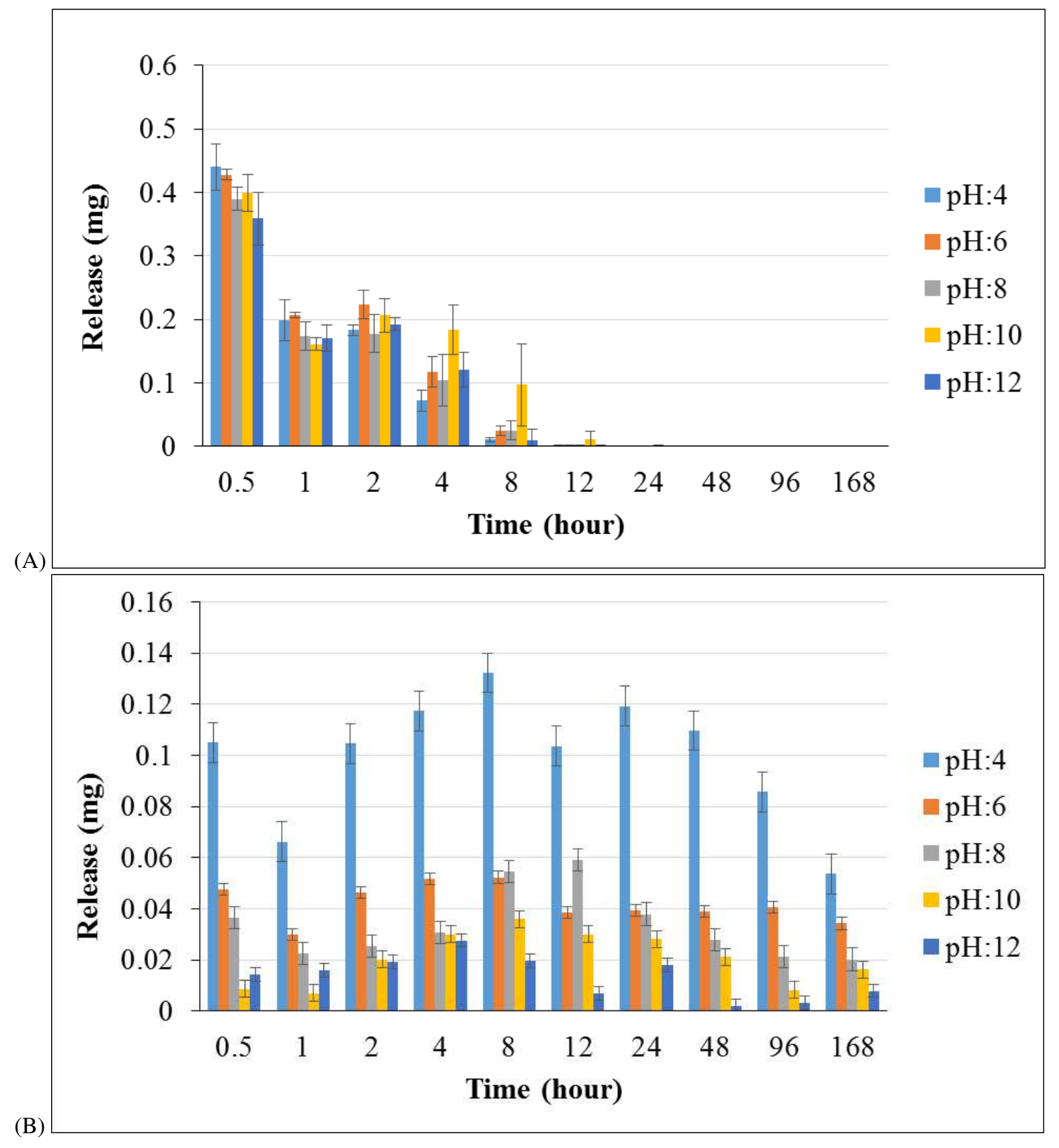

Figure 6. (A) Metr and (B) CHX release properties of NCHGs

In different $\mathrm{pH}$ Britton Robinson buffer solutions during one week period. Values are expressed as sample means, error bars represent the standard deviation (SD) of three parallel measurements. 


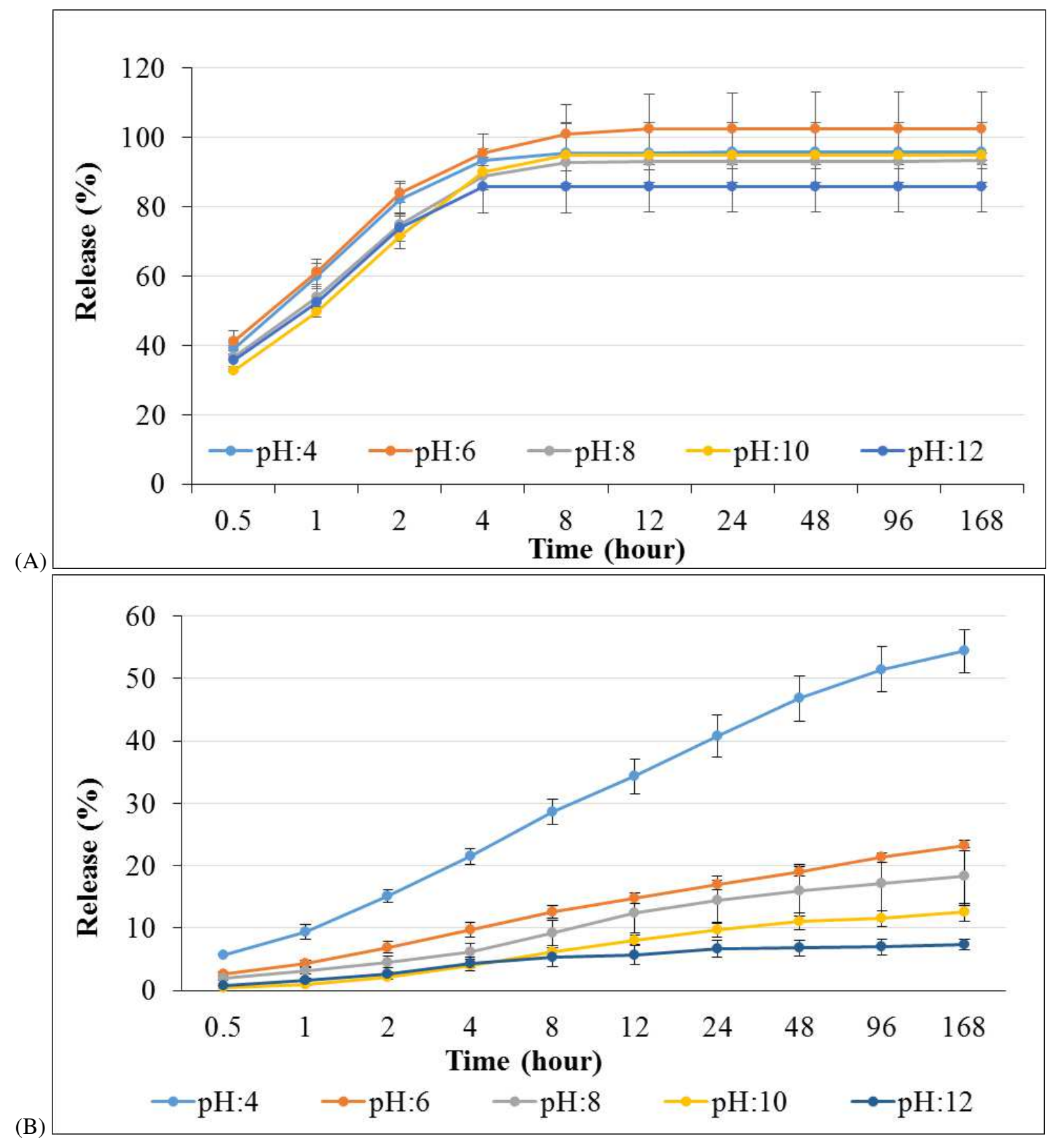

Figure 7. Presentation of cumulative amounts of released drugs for (A) Metr and (B) CHX data In different pHs Briton-Robinson buffer solutions by the accumulation of the released Metr (A) and CHX (B) in different measuring points and the relations to the entire drug contents. Values are expressed as sample means, error bars represent the standard deviation (SD) of three parallel measurements. 


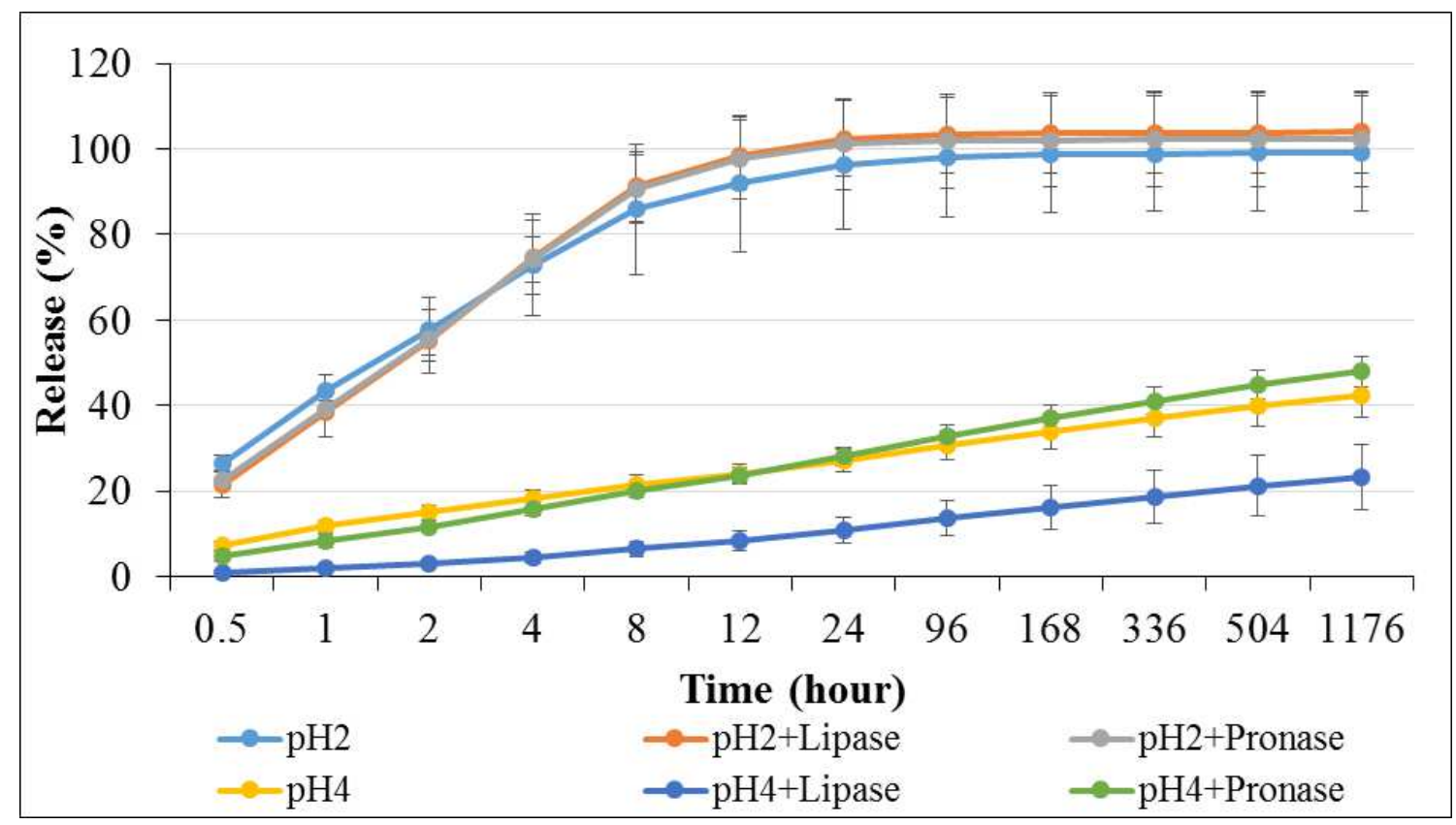

Figure 8. Presentation of cumulative amounts of released drugs

In different $\mathrm{pHs}$, and with different enzymes by the accumulation of the released CHX in different measuring points and the relations to the entire drug contents. Values are expressed as sample means, error bars represent the standard deviation (SD) of three parallel measurements.

\section{Viability staining on the hydrogel surface}

To investigate whether the cells can grow on the surface of the hydrogels, hydrogel layers were attached to glass coverslips which were placed into the wells of 24-well plates and MG63 cells were seeded onto the surface. Glass coverslips without hydrogels served as controls.

The aggregation of the cells was observed after 24 hours of seeding (see Figure 10) on the hydrogel samples, which became more prominent after 48 hours and reached almost total confluency at the $72^{\text {nd }}$ hour with the emergence of dead cell clusters, probably inside the origin of the cell aggregates. In contrast, the cells on the coverslips showed normal distribution, proliferation, and viability over the examination period (Figure 10). Therefore, we presume, that the hydrogel scaffold presented in this study is appropriate to provide a stable and viable environment for the cells surrounding the site of application.

\section{Antibiotic effect investigation}

On agar plates, the antibiotic effects of NCHG filled with Metr and CHX were compared to the solution of the same amount of drugs and the effect of the $\mathrm{pH} 2$ buffer solution for the release of the drugs was studied (see Table 2 and Figure 11). An antibiotic effect, similar to the control was observed at the drug-loaded NCHG, the circle of inhibition was a little bit smaller in the case of NCHG, but this can be caused by the retaining effect of the gel structure. The inhibition zones were grown to $14.67 \mathrm{~mm}$ from $12.54 \mathrm{~mm}$ at the solution from 4 to 24 hours, and the NCHG has shown an increase from $10.78 \mathrm{~mm}$ to 12.39 $\mathrm{mm}$. The effect of acidic buffer on the release was more prominent because after 4 hours it has shown a $24.20 \mathrm{~mm}$ diameter inhibition circle, which has not changed substantially after 24 hours $(23.13 \mathrm{~mm})$. Statistically, the results of antibacterial measures concerning the NCHG and NCHG with pH2 differed in the mean values significantly both for the 4 and the 24 hour measures according to the parametric $(\mathrm{t})$ and non-parametric (Mann-Whitney) tests $(\mathrm{P}<0.02$ in all cases). These results proved the notable effect of the $\mathrm{pH}$ of this combined drug delivery system and represent the more explicit effectiveness of the acidic environment e.g. in a closed volume, under an inflamed and sore gum.

\section{Material and methods}

\section{Modifications of PGA}

The poly- $\gamma$-glutamic acid (PGA, MW= $1.2 \times 106 \mathrm{Da}$, from GPC) was purchased from Nanjing Saitaisi Biotechnology Co. (Nanjing, China). Water-soluble 1-[3-(dimethylamino) propyl]-3-ethyl carbodiimide hydrochloride (EDC) (Carbosyth Limited, UK), 2,2'-(Ethylenedioxy)bis(ethylamine) (98\%) (EDA) (Sigma-Aldrich, USA) as a crosslinker and 2-aminoethyl methacrylate 


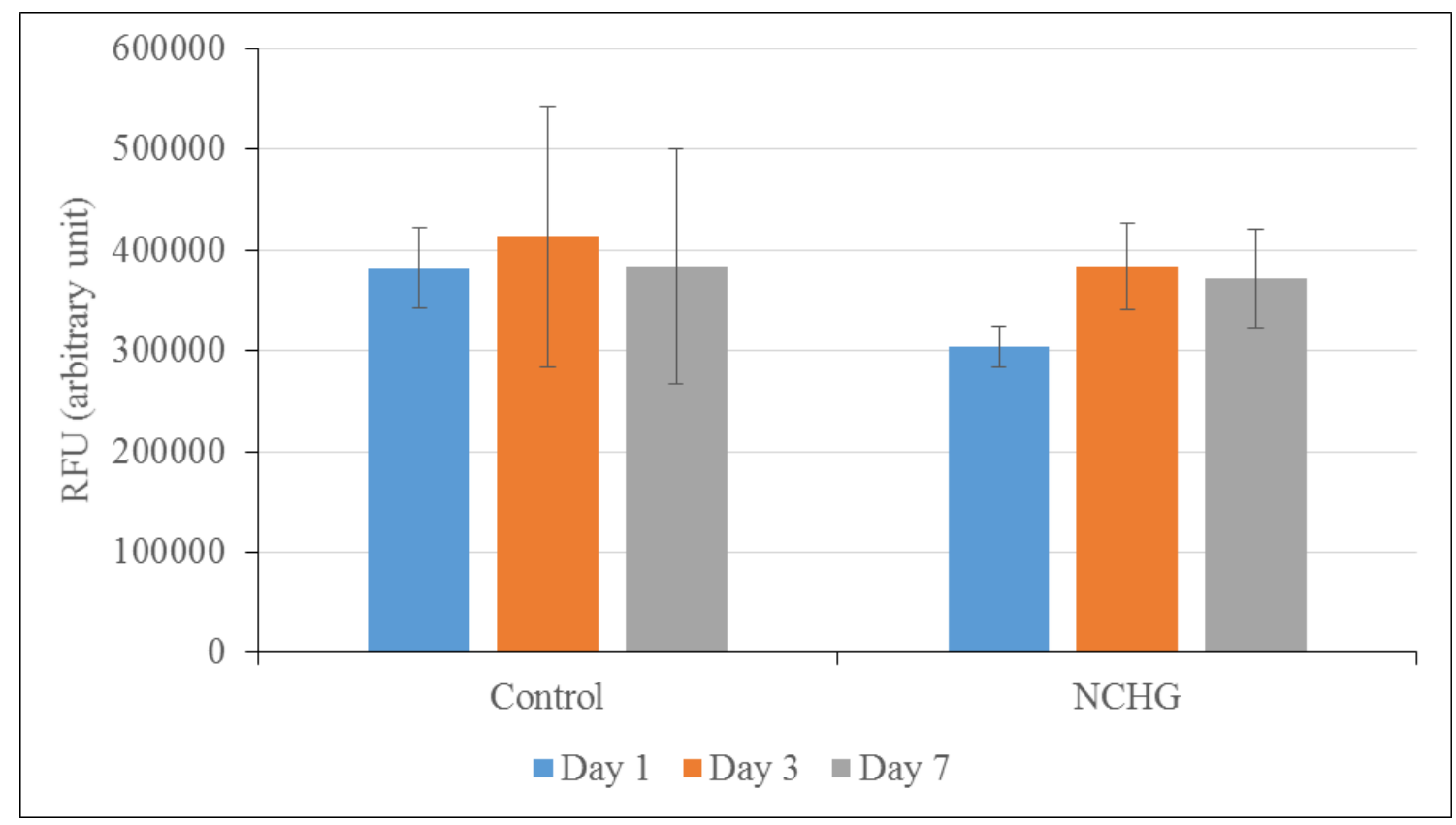

Figure 9. Cell viability assay of MG63 cells

Cells were cultured in the presence (NCHG) or absence (Control) of CHX and Metr containing hydrogel samples for 1, 3, and 7 days. After incubation, cell viability was measured by Alamar blue assay. Values are expressed as sample means, error bars represent the standard deviation (SD) of three parallel measurements.

\begin{tabular}{|c|c|c|c|c|}
\hline $\mathrm{n}=5$ & \multicolumn{2}{|c|}{ 4 hours } & \multicolumn{2}{c|}{24 hours } \\
\hline$(\mathrm{mm})$ & Mean & SD & Mean & SD \\
\hline \hline+ & 12.5394 & 0.6613 & 14.6773 & 0.9291 \\
NCHG & 10.7816 & 1.3406 & 12.3976 & 0.8360 \\
NCHG+ pH2 & 24.2077 & 3.1961 & 23.1351 & 2.8713 \\
\hline
\end{tabular}

Table 2. Diameters of inhibition zones in antibiotic investigations

hydrochloride (95\%) (AEM) (Polysciences Europe GmbH, Germay) as a methacrylating agent were applied. The methacrylatedpoly- $\gamma$-glutamic acid (MPGA) polymer was produced as we wrote it earlier 14. Briefly, PGA was activated by EDC, and methacryloyl-groups were created by AEM. Another component of NCHG, the methacrylated-poly- $\gamma$-glutamic acid nanoparticles (PGA-MNP) was made in a two steps reaction 13. Firstly, nanoparticles were formed. In this step, EDC was used for the increase of the reactivity of PGA, and EDA was used for the creation of crosslinked polymer which formed NPs. In the second step, the remaining carboxyl-groups were activated by a new portion of EDC, and the created PGA-NPs were modified by the methacryloyl-group used by AEM.

\section{Characterization of the methacrylated components}

${ }^{1} \mathrm{H}$ NMR spectra of the modified PGAs were recorded by Proton Nuclear Magnetic Resonance Spectroscopy $\left({ }^{1} \mathrm{H}\right.$ NMR) on a Bruker 200SY NMR spectrometer $(200 \mathrm{MHz})$ instrument. The samples were dissolved in deuterated water $\left(\mathrm{D}_{2} \mathrm{O}\right)$ and the chemical shifts were represented in parts per million ( $\mathrm{ppm}$ ) based on the signal of sodium 3-(trimethylsilyl)-propionate- $\mathrm{d}_{4}$ as a reference.

\section{Synthesis of nanocomposite hydrogel}

MPGA and PGA-MNP based nanocomposite hydrogels (NCHGs) were synthesized by free radical-initiated photopolymerization in solutions of antiseptic and antibiotic drugs. $22.2 \mathrm{w} / \mathrm{w} \%$ of MPGA and $11.1 \mathrm{w} / \mathrm{w} \%$ PGA-MNPs were mixed with the solution of Irgacure 2959 ( $99 \%$, Sigma-Aldrich, USA) as a photoinitiator ( $8 \mathrm{n} / \mathrm{n} \%$ calculated for methacryloyl-group), metronidazole (Metr) (98\%, Sigma-Aldrich, China), and chlorhexidine digluconate solution (CHX) (20\%, Sigma-Aldrich, Spain) as active substances. The photo-curing of the nanocomposite hydrogel samples was performed by a Bluephase $20 \mathrm{i}$ 

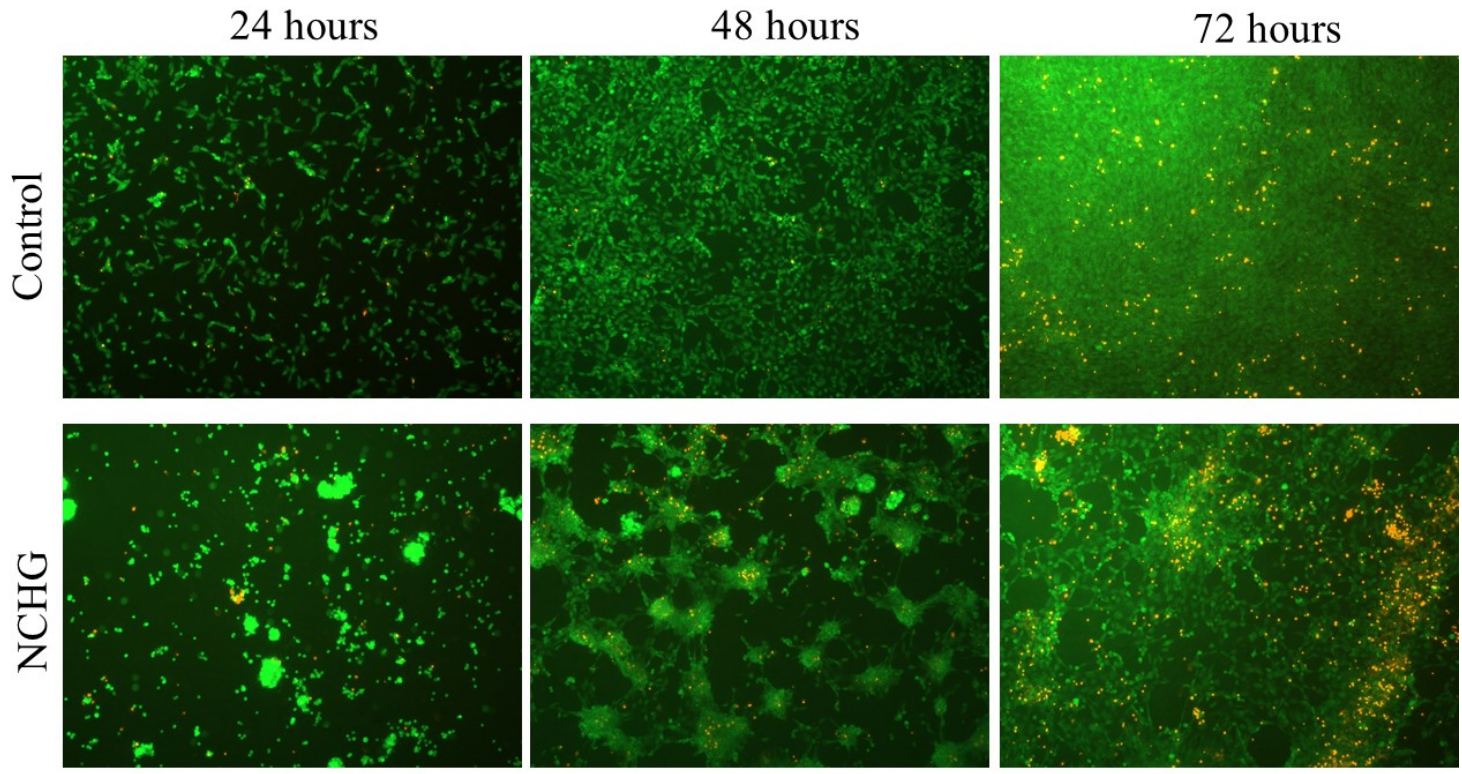

Figure 10. Vitality staining of MG63 cells

The cells were seeded onto hydrogel samples (NC HG) and untreated coverslips (Control) and cultured for 24, 48, and 72 hours. After the incubation period, cells on the different surfaces were co-stained by fluorescein diacetate and propidium iodide.

(Ivoclar Vivadent AG, Austria) dental polymerization unit (hand lamp). The samples were made in a $2 \mathrm{~mm}$ depth and $5 \mathrm{~mm}$ diameter cylindrical Teflon mold. The reaction times were $60 \mathrm{~s}$ with $2000 \mathrm{~mW} / \mathrm{cm}^{2}$.

\section{Characterization of the MPGA/PGA-NP nanocomposite hydrogel Mechanical investigations}

The mechanical properties of the NCHGs were investigated with an INSTRON 5544 Universal Mechanical Analyzer (Instron Inc, USA). The polymerizability and the effects of the loaded drugs for the main physical parameters were studied with the comparison of the compression test results of the drug-containing systems with the NCHGs without active ingredients. The studies were performed with a full-scale load range at $0.1 \mathrm{kN}$ and crosshead speed at $2 \mathrm{~mm} / \mathrm{min}$. The cylindrical hydrogel samples had a diameter of $5 \mathrm{~mm}$ and a specimen length of $2 \mathrm{~mm}$.

\section{Swelling properties}

The swelling parameters of the NCHGs were observed by gravimetric analysis. It was carried out by immersion of the samples in adjusted Universal Britton-Robinson buffer solutions (UB-RBS) ( $\mathrm{pH} 4-\mathrm{pH} 12)$. The buffer solutions were individually produced from boric-acid 99.5\% (Reanal, Hungary), phosphoric acid 85\% (VWR Chemicals, EC) and glacial acetic acid (VWR Chemicals, France), and were adjusted by $\mathrm{NaOH}$ solution (0.2 M) (VWR Chemicals, Belgium). The samples were removed from the media and wiped cautiously with bolting paper to eliminate the excess wetness from the surface. The measuring period was 168 hours. The weight swelling percentage $(\mathrm{Wp})$ for each sample was calculated as:

$$
\mathrm{Wp}=\frac{\mathrm{Ws}-\mathrm{Wo}}{\mathrm{Wo}} \times 100
$$

where Ws is the weight of the swollen gel and Wo is the original weight of the gel after polymerization.

\section{Study of drug release properties on different $\mathrm{pH}$}

The NCHGs were prepared for release studies with $50 \mathrm{mg} / \mathrm{g}$ CHX and $25 \mathrm{mg} / \mathrm{g}$ Metr content. The main purpose of these experiments was to examine the release rates of the drugs from the loaded hydrogels. The samples were immersed in an adjusted UB-RBS (1 ml) (pH $4-\mathrm{pH} 12)$ and subjected to continuous stirring on Heidolph Unimax 1010 plate shaker (Heidolph Instruments, Germany) (100 RPM). At predetermined periods the entire amount of medium (1 ml) was changed, and the concentrations of the drugs were measured by HPLC. A Dionex Ultimate 3000 (Dionex Softron GmbH, Germany) instrument with Hypersil Gold CN $(3 \mu \mathrm{m})$ column (Thermo Scientific, USA) was used and the absorbances were determined at $258 \mathrm{~nm}$ for 

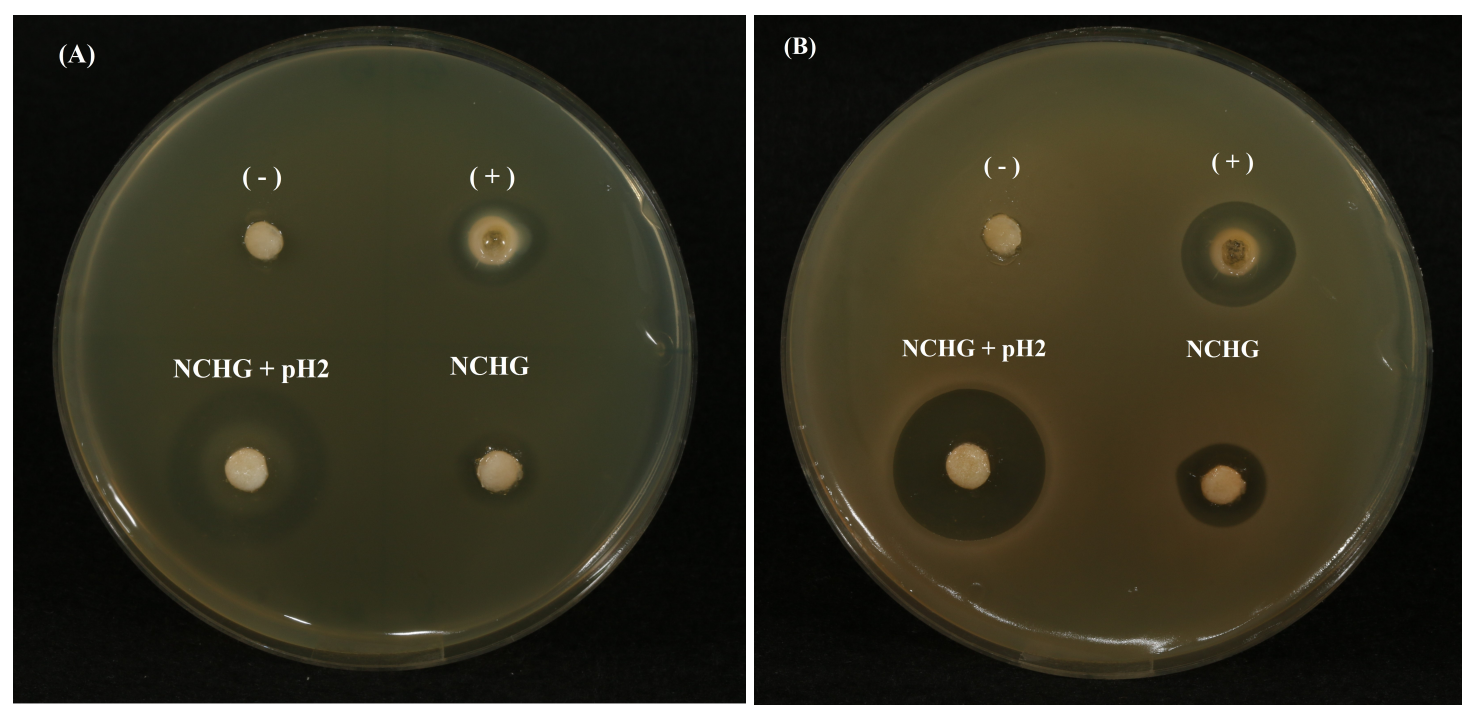

Figure 11. Antibiotic effect of $\mathrm{CHX} /$ metronidazole filled hydrogels

NCHG samples without drugs (-); metronidazole/CHX solution was used as a positive control (+); CHX/metronidazole containing NCHGs were investigated with distilled water (down on the right side), and with $\mathrm{pH} 2$ buffer solution (down and on the left side) (A) picture show the results on the 4th hour, and the (B) image represent the 24 hours effects. Samples were placed into agar plates inoculated with E. coli K12 ER2738. for the study of antibiotic effect.

CHX and $318 \mathrm{~nm}$ for Metr. The mobile phase was $70 \mathrm{w} / \mathrm{w} \%$ saline-solution (Fresenius Kabi, Germany) with $0.2 \mathrm{w} / \mathrm{w} \%$ formic acid (Sigma Aldrich, USA) and $30 \mathrm{w} / \mathrm{w} \%$ acetonitrile (VWR Chemicals, France), the flow rate was $0.6 \mathrm{ml} / \mathrm{min}$. The removed liquid was replaced by a freshly adjusted buffer solution. The amount of released drugs during the predetermined periods and the cumulative amount of drugs were expressed as the percentage of the original drug content. The determination of the release of the total amount of drugs happened using the same method, but $\mathrm{pH} 2$ buffer solutions, lipase (from hogpancrease $30.1 \mathrm{U} / \mathrm{mg}$ ) (Fluka, USA), and pronase (from Streptomyces griseus $5.05 \mathrm{U} / \mathrm{mg}$ ) (Fluka, Japan) were used additionally.

\section{Cell viability assay}

Human MG63 cell line (ATCC, USA) were cultured in Dulbecco's Minimum Essential Medium (Sigma-Aldrich, USA) containing $10 \%$ Fetal bovine serum, $1 \%$ Antibiotic-antimycotic solution, and $1 \%$ GlutaMAX (all from Gibco, Life Technologies, USA) at $37^{\circ} \mathrm{C}$ and $5 \% \mathrm{CO}_{2}$.

For Alamar Blue assay, $10^{5}$ cells/well were placed in a 24 -well cell culture plate and were let to attach for 24 hours. After attachment media were replaced with fresh medium, in which MPGA/PGA-MNP nanocomposite hydrogels ( $2 \mathrm{~mm} \times 5 \mathrm{~mm}$ gels were used) were submerged using Millipore 24 Well Millicell hanging cell culture inserts $0.4 \mu \mathrm{m}$ PET (Millipore Co. Billerica, $\mathrm{MA}$ ), and incubated at $37^{\circ} \mathrm{C}$ in a $\mathrm{CO}_{2}$ incubator. MG63 cells grown in the absence of hydrogel samples were used as control. After 1, 3, and 7 days media was replaced with 10 times diluted Alamar Blue reagent (Invitrogen, Life Technologies), then after 2 hours of incubation at $37^{\circ} \mathrm{C}$ and $5 \% \mathrm{CO}_{2}$ the fluorescence of the samples was measured using a microplate reader (HIDEX Sense, Finland). Hydrogels were removed before and were placed back after the measurements.

\section{Vitality staining}

The hydrogel samples for microscopic analyses were fixed chemically to glass surface. $13 \mathrm{~mm}$ diameter \#1.5 circle coverslips (Thermo Scientific, Germany) were treated with a 1:1 solution of $48 \mathrm{v} / \mathrm{v} \%$ hydrofluoric acid (VWR International, ECR) and distilled water for $1 \mathrm{~min}$, and after cleaning (twice in distilled water and once in acetone), they were modified with silane molecule (Ultradent ${ }^{\circledR}$ Silane, Ultradent Products Inc., USA). After air drying, the NCHG was applied as a thin layer and was chemically attached by $60 \mathrm{~s}$ of photopolymerization with a Bluephase $20 \mathrm{i}$ dental hand lamp. These samples were placed into a 24-well plate and were disinfected for 30 minutes by UV light.

In a 24-well plate, 105 MG63 cells/well were seeded onto the hydrogel samples, then incubated for 24, 48, and 72 hours. Untreated coverslips were used as a negative control. After the incubation period, the cells were co-stained with fluorescein diacetate (FDA) and propidium iodide (PI) (both from Sigma-Aldrich) for 5 minutes, at room temperature. Pictures were taken with Zeiss AxioVert A1 inverted fluorescence microscope (Zeiss, Germany). 


\section{Antibiotic release examination in agar plates}

$0.7 \%$ agar at $48{ }^{\circ} \mathrm{C}$ were inoculated with E. Coli K12 ER2738 in the mid-log phase, then mixed and poured into Petri dishes containing a layer of solid $1 \%$ agar and left to solidify at room temperature. NCHG hydrogel samples with or without Metr and CHX were placed into $5 \mathrm{~mm}$ diameter holes that were prepared into the agar plates. $10 \mu \mathrm{lpH} 2$ buffer solution was dropped to the Metr/CHX containing sample to study the acidic effect on the drug release. The $50 \mu 1 \mathrm{Metr} / \mathrm{CHX}$ solution was used as a positive control in the experiments. The plates were placed at $37^{\circ} \mathrm{C}$ and incubated for 4 and 24 hours. After the incubation periods, pictures were taken using a Canon EOS 70D (Canon, Japan) camera, and the diameter of the zone of inhibition was used to assess the release properties of the hydrogels.

\section{Statistical analysis}

All data are shown as mean \pm standard deviation (SD). Statistical analysis of viability tests was carried out using the Student's $\mathrm{t}$-test to determine the statistical significance of differences between of experimental groups. $\mathrm{P}<0.05$-issued to determine significance. GraphPad Prism v8 (GraphPad Software Inc., USA) was used for the investigations.

In the mechanical data the comparison of the means of the control and NCHG measures was performed by the independent sample $t$ test or the Welch $t$ test, depending on the equality of variances. The latter condition was checked by Levene's $F$ test. For all cases, furthermore, a non-parametric counterpart of the above tests, the Mann-Whitney was also run. The same tests were run for the comparison of the means of $\mathrm{NCHG}$ and $\mathrm{NCHG}$ with $+\mathrm{pH} 2$ medium samples groups regarding the antibacterial effect investigation.

To analyze the relationship between swelling (\%) and $\mathrm{pH}$ we fitted standard linear and non-linear regression models. Whereas the cumulative releases were explained by a multiple mixed effect regression model, for which the logit transform of the original release was used as dependent variable. The statistical calculations were done in IBM SPSS Statistics (Version 27, IBM, USA).

\section{Conclusion}

The photo-polymerizable MPGA and photo-reactive PGA-MNP created nanocomposite hydrogels show an alternative route of administration of different dental used drugs. This system can withstand the typical forces which can appear next to a tooth in an inflammatory situation. This photo-curable nanocomposite hydrogel ensures more than one-week antiseptic effect over a half a day higher concentration of antibiotic drug-eluting in a lower $\mathrm{pH}$, e.g. in a typical inflammatory situation locally. The biocompatible, and biodegradable stock materials and the $\mathrm{pH}$-dependent release properties together with the short-term blue light activation resulted in a practically useable and effective way against the bacteria colonies which cause periodontal inflammations because of two steps mechanism. This study presented a novel possibility of the curing of periodontitis in a comfortable, effective, and long-term manner.

\section{Acknowledgment}

The research was financed by the Thematic Excellence Programme 2020 (TKP2020-IKA-04) of the National Research, Development and Innovation Office, Hungary within the framework of the Biotechnology Thematic Programme of the University of Debrecen.

\section{Author contributions}

J.B. contributed to conception, design, data acquisition, analysis, interpretation and drafted the manuscript, F.T. contributed to data acquisition, analysis of molecular biological experiments, interpretation and drafted the manuscript, J.G. contributed to data acquisition, analysis and revised the manuscript, I.V. contributed to design, interpretation and critically revised the manuscript, A.S. contributed to conception, design, interpretation and critically revised the manuscript, R. Z. contributed to analysis, interpretation and critically revised the manuscript, C.H. contributed to conception, design, interpretation and critically revised the manuscript. All authors reviewed, edited and approved the manuscript for publication.

\section{Competing interests}

The authors declare no competing interests.

\section{Additional information}

Correspondence and requests for materials should be addressed to C.H or J.B. 


\section{References}

1. Emani, S., Gunjiganur, G. \& Mehta, D. Determination of the antibacterial activity of simvastatin against periodontal pathogens, porphyromonas gingivalis and aggregatibacter actinomycetemcomitans: an in vitro study. Contemporary Clinical Dentistry 5, 377-382, doi:10 . 4103/0976-237x.137959 (2014).

2. Nastri, L., De Rosa, A., De Gregorio, V., Grassia, V. \& Donnarumma, G. A new controlled-release material containing metronidazole and doxycycline for the treatment of periodontal and peri-implant diseases: formulation and in vitro testing. International Journal of Dentistry 2019, 9374607, doi:10 .1155/2019/9374607 (2019).

3. Albuquerque, M. T. P., Nagata, J. \& Bottino, M. C. Antimicrobial efficacy of triple antibiotic-eluting polymer nanofibers against multispecies biofilm. Journal of Endodontics 43, S51-S56, doi:10.1016/ j • joen.2017.06.009 (2017).

4. Rams, T. E. \& Slots, J. Local delivery of antimicrobial agents in the periodontal pocket. Periodontology, 2000 10, 139-159, doi:https://doi.org/10.1111/j.1600-0757.1996.tb00072.x(1996).

5. Chotitumnavee, J. et al. In vitro evaluation of local antibiotic delivery via fibrin hydrogel. Journal of Dental Sciences 14, 7-14, doi:https://doi.org/10.1016/j.jds.2018.08.010(2019).

6. Rajeshwari H. R. et al. Local drug delivery systems in the management of periodontitis: a scientific review. Journal of Controlled Release 307, 393-409, doi:https: / / doi.org/10.1016/j.jconrel.2019.06.038 (2019).

7. Hu, Y., Hu, S., Zhang, S., Dong, S., Hu, J., Kang, L. \& Yang, X. A double-layer hydrogel based on alginatecarboxymethyl cellulose and synthetic polymer as sustained drug delivery system. Scientific Reports 11, 9142, doi:10.1038/s41598-021-88503-1 (2021).

8. Hajishengallis, G., Chavakis, T. \& Lambris, J. D. Current understanding of periodontal disease pathogenesis and targets for host-modulation therapy. Periodontology 2000 84, 14-34, doi:https: / / doi.org/10.1111/prd.12331 (2020).

9. Slots, J. Primer on etiology and treatment of progressive/severe periodontitis: a systemic health perspective. Periodontology 2000 83, 272-276, doi:https: / / doi .org/10.1111/prd.12325 (2020).

10. Johnson, A. et al. Therapeutic effects of antibiotics loaded cellulose nanofiber and $\kappa$-carrageenan oligosaccharide composite hydrogels for periodontitis treatment. Scientific Reports 10,18037, doi:10.1038/s 41598-020-74845-9 (2020).

11. Pradeep, A. R., Sagar, S. V. \& Daisy, H. Clinical and microbiologic effects of subgingivally delivered $0.5 \%$ azithromycin in the treatment of chronic periodontitis. Journal of Periodontology 79, 2125-2135, doi:https://doi . org/10.1902/ jop.2008.070589(2008).

12. Lee, J. M., Jang, W. J., Park, S. H. \& Kong, I.-S. Antioxidant and gastrointestinal cytoprotective effect of edible polypeptide poly- $\gamma$-glutamic acid. International Journal of Biological Macromolecules 153, 616-624, doi:https: // doi . org/10. 1016/j.ijbiomac.2020.03.050(2020).

13. Bakó, J. et al. Poly- $\gamma$-glutamic acid nanoparticles based visible light-curable hydrogel for biomedical application. Journal of Nanomaterials 2016, 7350516, doi:10.1155/2016/7350516 (2016).

14. Bakó, J. et al. Composition and characterization of in situ usable light cured dental drug delivery hydrogel system. Journal of Materials Science: Materials in Medicine 24, 659-666, doi:10.1007/s10856-012-4825-x (2013).

15. Preshaw, P. M. Antibiotics in the treatment of periodontitis. Dent Update 31, 448-450, 453-444, 456, doi:10 . 12968 / denu.2004.31.8.448 (2004).

16. Pantlin, L. Is there a Role for Antibiotics in Periodontal Treatment? Dent Update 35, 493-496, doi:10.12968/denu . 2008.35 .7 .493 (2008).

17. Da Rocha, H. A. J. et al. Local drug delivery systems in the treatment of periodontitis: a literature review. J Int Acad Periodontol 17, 82-90 (2015).

18. Rafieian, S., Mirzadeh, H., Mahdavi, H. \& Masoumi, M. E. A review on nanocomposite hydrogels and their biomedical applications. Science and Engineering of Composite Materials 26, 154-174, doi:10.1515/secm-2017-0161 (2019).

19. Farjadian, F. et al. Temperature and $\mathrm{pH}$-responsive nano-hydrogel drug delivery system based on lysine-modified poly (vinylcaprolactam). Int J Nanomedicine 14, 6901-6915, doi:10 . 2147 / I JN. S21 4467 (2019).

20. Ainamo, J. et al. Clinical responses to subgingival application of a metronidazole $25 \%$ gel compared to the effect of subgingival scaling in adult periodontitis. Journal of Clinical Periodontology 19, 723-729, doi:https: // doi . org/10. 1111/j.1600-051X.1992.tb02535.x(1992).

21. Loesche, W. J. et al. Effects of metronidazole on periodontal treatment needs. Journal of Periodontology 62, 247-257, doi:https://doi.org/10.1902/jop.1991.62.4.247 (1991). 
22. Musial, W., Voncina, B., Pluta, J. \& Kokol, V. The study of release of chlorhexidine from preparations with modified thermosensitive poly-N-isopropylacrylamide microspheres. The Scientific World Journal 2012, 243707, doi:10.1100/ $2012 / 243707$ (2012).

23. Albayaty, Y. N. et al. Enzyme responsive copolymer micelles enhance the anti-biofilm efficacy of the antiseptic chlorhexidine. International Journal of Pharmaceutics 566, 329-341, doi:https://doi.org/10.1016/j.i jpharm.2019. 05.069 (2019).

24. Zeng, W. et al. Preparation and characterization of Poly ( $\gamma$-glutamic acid) hydrogels as potential tissue engineering scaffolds. Chinese Journal of Polymer Science 32, 1507-1514, doi:10.1007/s10118-014-1536-4 (2014).

25. Tronci, G., Grant, C. A., Thomson, N. H., Russell, S. J. \& Wood, D. J. Multi-scale mechanical characterization of highly swollen photo-activated collagen hydrogels. Journal of The Royal Society Interface 12, 20141079, doi:10.1098/rsif. 2014.1079 (2015).

26. Zhang, Q. et al. Adjustable and ultrafast light-cured hyaluronic acid hydrogel: promoting biocompatibility and cell growth. Journal of Materials Chemistry B 8, 5441-5450, doi:10 .1039/C9TB02796C (2020).

27. Greenstein, G. Local drug delivery in the treatment of periodontal diseases: assessing the clinical significance of the results. Journal of Periodontology 77, 565-578, doi:https://doi.org/10.1902/ jop.2006.050140 (2006).

28. Budai-Szúcs, M. et al. Electrospun Scaffolds in Periodontal Wound Healing. Polymers (Basel) 13, doi:10.3390/ polym13020307 (2021).

29. Killoy, W. J. The use of locally-delivered chlorhexidine in the treatment of periodontitis. Clinical results. Journal of Clinical Periodontology 25, 953-958, doi:https://doi.org/10.1111/j.1600-051X.1998.tb02397.x (1998).

30. Jepsen, K. \& Jepsen, S. Antibiotics/antimicrobials: systemic and local administration in the therapy of mild to moderately advanced periodontitis. Periodontology 2000 71, 82-112, doi:https://doi.org/10.1111/prd.12121 (2016).

31. Lang, N. P., Catalanotto, F. A., Knöpfli, R. U. \& Antczak, A. A. A. Quality-specific taste impairment following the application of chlorhexidine digluconate mouthrinses. Journal of Clinical Periodontology 15, 43-48, doi:https: //doi . org/10.1111/j.1600-051X.1988.tb01553.x(1988).

32. Gendron, R., Grenier, D., Sorsa, T. \& Mayrand, D. Inhibition of the activities of matrix metalloproteinases 2,8 , and 9 by chlorhexidine. Clinical Diagnostic Laboratory Immunology 6, 437-439, doi:10 .1128/CDLI . 6.3 .437-439.1999 (1999).

33. Grenier, D. Effect of chlorhexidine on the adherence properties of porphyromonas gingivalis. Journal of Clinical Periodontology 23, 140-142, doi:https://doi.org/10.1111/j.1600-051x.1996.tb00547.x(1996).

34. Pietruska, M., Paniczko, A., Waszkiel, D., Pietruski, J. \& Bernaczyk, A. Efficacy of local treatment with chlorhexidine gluconate drugs on the clinical status of periodontium in chronic periodontitis patients. Adv Med Sci 51 Suppl 1, 162-165 (2006).

35. Phaechamud, T. \& Setthajindalert, O. Cholesterol in situ forming gel loaded with doxycycline hyclate for intra-periodontal pocket delivery. Eur J Pharm Sci 99, 258-265, doi:10.1016/ j.ejps.2016.12.023 (2017).

36. Purucker, P., Mertes, H., Goodson, J. M. \& Bernimoulin, J.-P. Local versus systemic adjunctive antibiotic therapy in 28 patients with generalized aggressive periodontitis. Journal of Periodontology 72, 1241-1245, doi:https: // doi.org/ $10.1902 /$ jop.2000.72.9.1241(2001).

37. Tan, O. L., Safii, S. H. \& Razali, M. Commercial local pharmacotherapeutics and adjunctive agents for nonsurgical treatment of periodontitis: a contemporary review of clinical efficacies and challenges. Antibiotics 9, 11, doi: 10.3390/ antibiotics 9010011 (2020).

38. Steinberg, D. \& Friedman, M. Sustained-release delivery of antimicrobial drugs for the treatment of periodontal diseases: fantasy or already reality? Periodontology 2000 84, 176-187, doi:https://doi.org/10.1111/prd. 12341 (2020).

39. Chen, Y. C., Gad, S. F., Chobisa, D., Li, Y. \& Yeo, Y. Local drug delivery systems for inflammatory diseases: status quo, challenges, and opportunities. Journal of Controlled Release 330, 438-460, doi:https://doi.org/10.1016/j. jconrel.2020.12.025(2021).

40. Alvarez-Lorenzo, C., Grinberg, V. Y., Burova, T. V. \& Concheiro, A. Stimuli-sensitive cross-linked hydrogels as drug delivery systems: Impact of the drug on the responsiveness. International journal of pharmaceutics 579, 119157, doi:10 . 1016/j.ijpharm.2020.119157 (2020).

41. Wang, B. et al. A tunable and injectable local drug delivery system for personalized periodontal application. Journal of Controlled Release 324, 134-145, doi:https://doi.org/10.1016/j.jconrel.2020.05.004 (2020). 
42. Hu, W., Wang, Z., Xiao, Y., Zhang, S. \& Wang, J. Advances in crosslinking strategies of biomedical hydrogels. Biomaterials Science 7, 843-855, doi:10.1039/C8BM01246F (2019).

43. Vidal-Romero, G. et al. Design and evaluation of $\mathrm{pH}$-dependent nanosystems based on cellulose acetate phthalate, nanoparticles loaded with chlorhexidine for periodontal treatment. Pharmaceutics 11, 604, doi: 10.3390/ pharmaceutics11110604 (2019).

44. Hu, C., Zhang, F., Long, L., Kong, Q., Luo, R. \& Wang Y. Dual-responsive injectable hydrogels encapsulating drug-loaded micelles for on-demand antimicrobial activity and accelerated wound healing. Journal of Controlled Release 324, 204-217, doi:https://doi.org/10.1016/j.jconrel.2020.05.010 (2020).

45. Akram, Z., Aati, S., Ngo, H. \& Fawzy, A. pH-dependent delivery of chlorhexidine from PGA grafted mesoporous silica nanoparticles at resin-dentin interface. Journal of Nanobiotechnology 19, 43, doi:10.1186/s12951-021-00788-6 (2021).

46. Fullriede, $\mathrm{H}$. et al. pH-responsive release of chlorhexidine from modified nanoporous silica nanoparticles for dental applications. BioNanoMaterials 17, 59-72, doi:10.1515/bnm-2016-0003 (2016).

47. $\mathrm{Xu}, \mathrm{X}$. et al. An injectable and thermosensitive hydrogel: promoting periodontal regeneration by controlled-release of aspirin and erythropoietin. Acta Biomaterialia 86, 235-246, doi:https://doi .org/10.1016/j .actbio.2019.01.001 (2019).

48. Batool, F. et al. In-situ forming implants loaded with chlorhexidine and ibuprofen for periodontal treatment: proof of concept study in vivo. International Journal of Pharmaceutics 569, 118564, doi:https://doi.org/10.1016/j. ijpharm.2019.118564 (2019). 